\title{
Adiabatic high degree modes of a rotating star ${ }^{\star}$ I. General features and real pressure modes
}

\author{
F. Schmitz ${ }^{1}$ and B. Fleck ${ }^{2}$ \\ 1 Institut für Theoretische Physik und Astrophysik der Universität Würzburg, Am Hubland, 97074 Würzburg, Germany \\ e-mail: schmitz@astro.uni-wuerzburg.de \\ 2 ESA Sciencie Operations Department, c/o NASA/GSFC, Mailcode 671.1, Greenbelt, MD 20771, USA \\ e-mail: bfleck@esa.nascom.nasa.gov
}

Received 4 June 2009 / Accepted 2 March 2010

ABSTRACT

\begin{abstract}
Aims. The influence of the rotation of the Sun on non-radial $p$-modes with high wave numbers $l$ is studied. To investigate and understand the basic properties of these modes, it is sufficient to consider only the outer layers of the Sun, which can be approximated by a plane layer with constant gravity.

Methods. We use a model with a smooth transition between a polytropic convection zone and an isothermal atmosphere. The rotation is simulated by a constant horizontal wind. For this model, using the column mass instead of the geometrical height, the adiabatic wave equation of the pressure perturbation can be reduced to Whittaker's differential equation. From boundary conditions we obtain the dispersion relation. The geometrical height is a simple elementary function of the column mass.

Results. The dispersion relation $F(\omega, k)=0$ is a higher order algebraic equation in both frequency and horizontal wave number, which must be solved numerically. We analyze the behavior of the dispersion curves of modes with an adiabatic exponent $\gamma=5 / 3$ for layers with polytropic indices $n=3$ and $n=3 / 2$. The f-mode is considered separately. For the understanding of the results we also consider modes of a homogeneous gas. We compare the $k-\omega$ diagram of our idealized model with the $k-\omega$ diagram of a real solar
\end{abstract} model.

Key words. hydrodynamics - waves - stars: oscillations - Sun: oscillations - stars: atmospheres

\section{Introduction}

To study basic properties of solar p-modes with $l \gg 1$, it is sufficient to consider only the upper convection zone and the atmospheric layers of the Sun. For $l \gg 1$, the approach of these regions of the Sun by a plane layer with constant gravity is common. A simple model of the convection zone is a polytropic layer with a positive polytropic index $n$. In this case, the pressure and the temperature vanish at some height. The solution of the adiabatic wave equation of this layer has to satisfy a zero pressure boundary condition. By the assumption of vanishing pressure perturbations in the interior, a discrete spectrum of modes is obtained. This problem was already investigated by Lamb (1932). The isolated polytropic convection zone corresponds to simple polytropic stellar models with zero pressure boundary condition and moderate central densities, as far as Cowling's classification is valid.

We use the same procedure as in the windless case studied by Schmitz \& Steffens (1999). The model consists of a convection zone and an atmosphere, which are fitted by a smooth temperature transition. The convection zone becomes polytropic as $z \rightarrow-\infty$, the atmosphere becomes isothermal as $z \rightarrow+\infty$.

In Sect. 2 we discuss previous works on oscillations of rotating stars. Section 3 deals with the structure of the static layer. In Sect. 4 we present the adiabatic wave equation. The wave equation of the Lagrangian pressure perturbation is formulated

^ Dedicated to Franz-Ludwig Deubner, who celebrated his 75th birthday on June 2, 2009. in terms of the column mass. Section 5 deals with the reduction of the wave equation to Whittaker's differential equation. The general solution of this equation is considered in Sect. 6. The dispersion relation of the modes of the layer, an algebraic equation of a higher order in $\omega^{2}$ and the horizontal wave numbers is derived in Sect. 7. It is solved numerically. In Sect. 8 we consider the f-mode, and in Sect. 9 we discuss oscillations of a homogenous gas. In Sect. 10 we present dispersion curves for the Sun in the non-isentropic case $\gamma=5 / 3$ and $1+1 / n=4 / 3$ and for the isentropic case $\gamma=1+1 / n=5 / 3$.

\section{Oscillations of rotating stars}

There are numerous text-books (e.g. Tassoul 1978), compendia (e.g. Ledoux \& Walraven 1958), review-articles (e.g. Saio 1993) and publications on the field of oscillations and the stability of rotating stars (e.g. Lynden-Bell 1967) and the stability of gaseous clouds (e.g. Schmitz 1984).

As regards the Sun, it is now nearly 35 years since Deubner's observations of low wavenumber non-radial acoustic eigenmodes (Deubner 1975) that launched the art and science of helioseismology and led to the first determination of the radial differential roation of the Sun (Deubner 1979, see also review by Deubner \& Gough 1984).

Schou et al. (1998) used the splitting of the frequencies of the global resonant acoustic modes induced by large-scale flows and rotation to determine the Sun's interior angular velocity. In all these works a zero-pressure boundary condition is assumed, i.e. the pressure becomes zero at the stellar radius. With increasing 
$l$, the region of the oscillation shifts outwards into the outer convection zone and the atmosphere. Thus for high degree-modes, the detailed structure of this layer must be taken into account. For stars without rotation this was done e.g. by ChristensenDaalsgard (1980) and for the Sun by e.g. Ando \& Osaki (1977).

The model of Schmitz \& Steffens (1999) is a simple approach to the outer layers of the Sun, far from the real models with ionization and dissoziation considered by Antja \& Basu (1999) and Steffens \& Schmitz (2000).

However, it does not make sense to consider the detailed structure of the outer layers. The VAL-atmosphere, often used as a standard atmosphere, is not realistic. Calculations in particular by Carlson \& Stein (1995) have shown that the atmosphere is so dynamic that it is not possible to assign a mean temperature to the chromosphere.

For these reasons Steffens and Schmitz studied the influence of cool-temperature and mean-temperature "chromospheres" on solar oscillations.

Here we present an analytical study of the influence of solar rotation on the p-mode ridges. We use the model of Schmitz \& Steffens (1999) with a horizontal constant wind. The continuos wave number $k_{x}$ in the direction of the wind corresponds to the discrete wave number $m$, the continuos wave number $k_{y}$ corresponds to the discrete wave number $l$.

\section{The equilibrium layer}

Let $z$ be the vertical, outwards directed geometrical coordinate, $g$ the constant gravity, $n$ the polytropic index, $m$ the column mass, defined by $\mathrm{d} m=-\rho \mathrm{d} z, p$ the pressure, $\rho$ the density, $a$ the isothermal and $c$ the adiabatic sound speed, $\gamma$ the adiabatic exponent. We use the equation of state of the classical ideal gas $p=a^{2} \rho$. The atmospheric structure is not influenced by the horizontal wind. The equilibrium condition is $p=m g$. We put

$c^{2}(m)=c_{0}^{2}+\epsilon m^{\lambda}$

with $\lambda=1 /(1+n)$. For the Sun, the parameter $\epsilon$ is $\approx 10^{11}$ in cgs-units. For $m \rightarrow 0$ the layer becomes isothermal with $c=c_{0}$, for large $m$ the layer becomes polytropic with the index $n$. In the limit $c_{0}=0$ we obtain the polytropic layer. The density is

$\rho(m)=\frac{m g}{c_{0}^{2} / \gamma+\epsilon m^{\lambda}}$.

The geometrical height $z(m)$ is obtained by integrating $\mathrm{d} z=$ $-\mathrm{d} m / \rho$. We get

$z=-\frac{1}{g}\left[\frac{c_{0}^{2}}{\gamma} \ln m+\frac{\epsilon}{\lambda} m^{\lambda}\right]$.

We have $m \rightarrow 0$ as $z \rightarrow+\infty$ and $m \rightarrow \infty$ as $z \rightarrow-\infty$, and

$c^{2}(z)=-\frac{\gamma g}{1+n} z$ for $z \rightarrow-\infty$.

\section{The adiabatic wave equation}

Let $\Delta p(z, t)$ be the Lagrangian pressure perturbation, $\xi(z, t)$ the displacement. The frequency is denoted by $\omega$, the horizontal wave numbers by $k_{x}$ and $k_{y}$. We put

$k^{2}=k_{x}^{2}+k_{y}^{2}$.

We study adiabatic waves with time dependence $\exp (\mathrm{i} \omega t)$. We assume that the wind velocity $v$ is constant. We put

$\Omega=\omega-v k_{x}$.
From the linearized hydrodynamical equations we obtain two first order equations:

$\Omega \omega \frac{\mathrm{d} \Delta p}{\mathrm{~d} z}+g k^{2} \Delta p=\rho\left[\Omega^{2} \omega^{2}-g^{2} k^{2}\right] \xi$

and

$\Omega \omega \rho c^{2} \frac{\mathrm{d} \xi}{\mathrm{d} z}-\rho g k^{2} c^{2} \xi=-\left[\omega^{2}-k^{2} c^{2}-v k_{x} \omega\right] \Delta p$

(see, e.g. Schmitz \& Fleck 1994).

From these equations we obtain the wave equation of the Lagrangian pressure perturbation $\Delta p$ :

$\Omega^{2} \omega \frac{\mathrm{d}^{2} \Delta p}{\mathrm{~d} z^{2}}$

$+\left[\Omega^{2} g k^{2} \frac{1}{\omega}-\left[\rho k^{2} g \frac{1}{\omega}+\frac{\mathrm{d} \rho}{\mathrm{d} z} \Omega\right] \frac{1}{\rho} \Omega \omega\right] \frac{\mathrm{d} \Delta p}{\mathrm{~d} z}$

$+\left[\left[\Omega^{2} \omega^{2}-g^{2} k^{2}\right] \frac{1}{\omega c^{2}}\left[\omega^{2}-k^{2} c^{2}-v k_{x} \omega\right]\right.$

$\left.-\left[\rho k^{2} g \frac{1}{\omega}+\frac{\mathrm{d} \rho}{\mathrm{d} z} \Omega\right] \frac{1}{\rho} g k^{2}\right] \Delta p=0$,

and the wave equation of the displacement $\xi$ :

$\Omega^{2}\left[\Omega^{2}-k^{2} c^{2}\right] c^{2} \frac{d^{2} \xi}{d z^{2}}$

$+\Omega^{2}\left[-\gamma g\left[\Omega^{2}-k^{2} c^{2}\right]+k^{2} c^{2} \frac{\mathrm{d} c^{2}}{\mathrm{~d} z}\right] \frac{\mathrm{d} \xi}{\mathrm{d} z}$

$+\left[k^{2}\left[\Omega^{2}-k^{2} c^{2}\right]\left[(\gamma-1) g^{2}+g \frac{\mathrm{d} c^{2}}{\mathrm{~d} z}\right]\right.$

$\left.+\Omega^{2}\left[\Omega^{2}-k^{2} c^{2}\right]^{2}-\Omega^{2} k^{2} g \frac{\mathrm{d} c^{2}}{\mathrm{~d} z}\right] \xi=0$.

The wave equation of $\Delta p$ has an absorption level, the equation of $\xi$ an additional reflection level. Therefore we use the equation of $\Delta p$. Beer (1975) has studied the behavior of waves in an isothermal atmosphere with constant wind by the wave equation of the displacement.

Now we take the mass $m$ instead of the height $z$. We have

$\frac{\mathrm{d}}{\mathrm{d} z}=-\rho \frac{\mathrm{d}}{\mathrm{d} m}$

and

$\frac{\mathrm{d}^{2}}{\mathrm{~d} z^{2}}=\frac{g^{2} \gamma^{2} m^{2}}{c^{4}} \frac{\mathrm{d}^{2}}{\mathrm{~d} m^{2}}+\frac{g^{2} \gamma^{2} m}{c^{4}}\left[1-\frac{m}{c^{2}} \frac{\mathrm{d}}{\mathrm{d} m} c^{2}\right] \frac{\mathrm{d}}{\mathrm{d} m}$.

Further,

$\frac{\mathrm{d}}{\mathrm{d} z} \rho=-\frac{\gamma g \rho}{c^{2}}\left[1-\frac{m}{c^{2}} \frac{\mathrm{d}}{\mathrm{d} m} c^{2}\right]$.

We finally obtain

$\frac{\mathrm{d}^{2} \Delta p}{\mathrm{~d} m^{2}}+\left(A+B m^{\lambda}\right) m \frac{\mathrm{d} \Delta p}{\mathrm{~d} m}$

$+\left(C++D m^{\lambda}+E m^{2 \lambda}\right) \Delta p=0$ 
F. Schmitz and B. Fleck: Adiabatic high degree modes of a rotating star. I.

with

$A=-\frac{k^{2} k_{x} v c^{2}}{\Omega \omega^{2} \gamma}$

$B=-\frac{k^{2} k_{x} v}{\Omega \omega^{2} \gamma} \epsilon$

$C=\left[\Omega^{2} \omega^{2}+(\gamma-1) g^{2} k^{2}-\Omega \omega k^{2} c^{2}\right] \frac{c^{2}}{\Omega \omega g^{2} \gamma^{2}}$,

$D=\left[\Omega^{2} \omega^{2}+((-\lambda+1) \gamma-1) g^{2} k^{2}-2 \Omega \omega k^{2} c^{2}\right]$

$\times \frac{\epsilon}{\Omega \omega g^{2} \gamma^{2}}$.

$E=-\frac{k^{2} \epsilon^{2}}{g^{2} \gamma^{2}}$

\section{The reduction of the adiabatic wave equation}

We put

$\Delta p=\xi^{v} \eta(\xi) \quad$ with $\quad \xi=m^{\lambda}$.

With these transformations we get

$\lambda^{2} \xi^{2} \frac{\mathrm{d}^{2} \eta}{\mathrm{d} \xi^{2}}+\left[(\lambda-1+A+2 v) \lambda \xi+B \lambda \xi^{2}\right] \frac{\mathrm{d} \eta}{\mathrm{d} \xi}$

$+\left[v \lambda(v \lambda+A-1)+C+(B \lambda v+D) \xi+E \xi^{2}\right] \eta=0$.

We put

$v^{2} \lambda^{2}+(A-1) \lambda v+C=0$,

which gives

$v=\frac{1}{2 \lambda^{2}}\left[-(A-1) \lambda \pm \sqrt{(A-1)^{2} \lambda^{2}-4 \lambda^{2} C}\right]$

Equation (22) reduces to

$$
\begin{aligned}
& \lambda^{2} \xi \frac{\mathrm{d}^{2} \eta}{\mathrm{d} \xi^{2}}+\left[\lambda B \xi+2 v \lambda^{2}+\lambda(\lambda-1+A)\right] \frac{\mathrm{d} \eta}{\mathrm{d} \xi} \\
& +[E \xi+v \lambda B+D]=0 .
\end{aligned}
$$

Now we put

$\eta=\xi^{-\beta / 2} \exp (-\alpha \xi / 2) w(\xi)$.

With this transformation we get

$$
\begin{aligned}
& \xi^{2} \frac{\mathrm{d}^{2} w}{\mathrm{~d} \xi^{2}}+\left[\left(\delta-\frac{\alpha^{2}}{4}\right) \xi^{2}\right. \\
& \left.+\left(\mathrm{d}-\frac{\alpha \beta}{2}\right) \xi+\frac{\beta}{2}\left(1-\frac{\beta}{2}\right)\right] w=0,
\end{aligned}
$$

where

$$
\begin{aligned}
& \beta=\frac{\lambda-1}{\lambda}+2 v+\frac{A}{\lambda}, \quad \alpha=\frac{B}{\lambda}, \\
& \mathrm{d}=\frac{B v}{\lambda}+\frac{D}{\lambda^{2}}, \quad \delta=\frac{E}{\lambda^{2}} .
\end{aligned}
$$

Putting

$\xi=\Lambda \zeta$

with

$$
\Lambda=\frac{1}{2 \sqrt{-\delta+\frac{\alpha^{2}}{4}}}
$$

we obtain the equation

$$
\begin{aligned}
& \frac{\mathrm{d}^{2} w}{\mathrm{~d} \zeta^{2}}+\left[-\frac{1}{4}+(d-\alpha \beta) \frac{\Lambda}{\zeta}\right. \\
& \left.+\left[\frac{1}{4}-\left(\frac{1}{4}-\frac{\beta}{2}\left(1-\frac{\beta}{2}\right)\right)\right] \frac{1}{\zeta^{2}}\right] w=0 .
\end{aligned}
$$

This is Whittaker's equation

$\frac{\mathrm{d}^{2} w}{\mathrm{~d} x^{2}}+\left[-\frac{1}{4}+\frac{\kappa}{x}+\left(\frac{1}{4}-\mu^{2}\right) \frac{1}{x^{2}}\right] w=0$

with

$\kappa=(\mathrm{d}-\alpha \beta) \Lambda$

and

$\left.\mu^{2}=-\frac{1}{4}+\frac{\beta}{2}\left(1-\frac{\beta}{2}\right)\right)$.

Finally,

$\mu^{2}=\frac{1}{4 \lambda^{2}}-\frac{v}{\lambda}+v^{2}-\frac{A}{2 \lambda}+\left(\frac{A}{2 \lambda}\right)^{2}$

and

$\kappa=\frac{1}{\lambda}\left[\frac{D}{\lambda}-\frac{A B}{2 \lambda}-B\left[\frac{1}{2}-\frac{1}{2 \lambda}\right]\right] \Lambda$.

Now let us assume that $k \neq 0$. For real $\omega^{2}$, the coefficient $\kappa$ is real, $\mu$ is real or imaginary.

\section{The solution of the wave equation}

Two independent solutions of Whittaker's equation are

$w_{1}=\mathrm{e}^{-\zeta / 2} \zeta^{1 / 2+\mu} M\left(\frac{1}{2}+\mu-\kappa, 1+2 \mu, \zeta\right)$,

$w_{2}=\mathrm{e}^{-\zeta / 2} \zeta^{1 / 2+\mu} U\left(\frac{1}{2}+\mu-\kappa, 1+2 \mu, \zeta\right)$,

where $M$ and $U$ are the confluent hypergeometric functions. The corresponding Lagrangian pressure perturbations are

$\Delta p_{1}=c_{1} m^{1 / 2+\lambda \mu} m^{-A / 2} \mathrm{e}^{-\zeta / 2-\alpha \xi / 2}$

$M\left(\frac{1}{2}+\mu-\kappa, 1+2 \mu, \zeta\right)$

and

$\Delta p_{2}=c_{2} m^{1 / 2+\lambda \mu} m^{-A / 2} \mathrm{e}^{-\zeta / 2-\alpha \xi / 2}$

$U\left(\frac{1}{2}+\mu-\kappa, 1+2 \mu, \zeta\right)$ 
where

$\zeta=\frac{1}{\Lambda} m^{\lambda}$

The general solution of the wave equation is a superposition of both solutions. The factor $m^{1 / 2} m^{\lambda \mu}$ is due to the isothermal atmosphere. In the limit $m \rightarrow 0$ where $z \rightarrow \infty$ we obtain

$m^{1 / 2} m^{\lambda \mu}=\exp \left(-\frac{z}{2 H}+\frac{\lambda \mu z}{H}\right)$ with $H=\frac{c_{0}^{2}}{\gamma g}$.

In the following, instead of $\omega^{2}$, we use the quantity $y$ defined by

$y=\frac{\omega^{2}}{g k}$

and instead of the wave number $k$ we use the relative wave number $k / k_{0}$ with

$k_{0}=\frac{g}{4 c_{0}^{2} \gamma}$.

\section{The dispersion relation of the modes}

For real $\mu$ we have to select the solution $\Delta p_{1}$ with $\mu>0$. Therefore,

$\Delta p \propto \zeta^{v+1 / 2+\mu} \mathrm{e}^{-\zeta / 2} M\left(\frac{1}{2}+\mu-\kappa, 1+2 \mu, \zeta\right)$.

As $M=1$ for $z=0$, this solution approaches the evanescently decaying wave of the unbounded isothermal atmosphere for $z \rightarrow$ $+\infty$ when $\mu>0$.

For $z \rightarrow-\infty$ or $x \rightarrow+\infty$ we require vanishing or at least finite pressure perturbations. This assumption is common and corresponds to the condition of vanishing non-radial pressure perturbations in the center of a star. 1965)

The asymptotic expansion of $M$ is (Abramowitz \& Stegun

$M(a, b, \zeta)=\frac{\Gamma(b)}{\Gamma(a)} \mathrm{e}^{\zeta} \zeta^{a-b}$ for $\zeta \rightarrow \infty$.

We obtain

$\Delta p \propto x^{\nu-\kappa} \mathrm{e}^{x / 2} \frac{\Gamma(1+2 \mu)}{\Gamma\left(\frac{1}{2}+\mu-\kappa\right)}$ for $x \rightarrow \infty$.

Therefore, the criterion for convergence at $\zeta \rightarrow \infty$ is

$\frac{1}{2}+\mu-\kappa=-j, \quad j=0,1,2,3 \ldots$

In this case, the function $M$ is reduced to a polynomial of degree $j$, a generalized Laguerre-polynomial. The pressure perturbation decays exponentially for $\zeta \rightarrow \infty$. Otherwise, the pressure perturbation diverges. Taking the square of

$\mu=\kappa-j-\frac{1}{2}$

we obtain

$\mu^{2}-\kappa^{2}+2 \kappa\left(j+\frac{1}{2}\right)-\left(j+\frac{1}{2}\right)^{2}=0$.

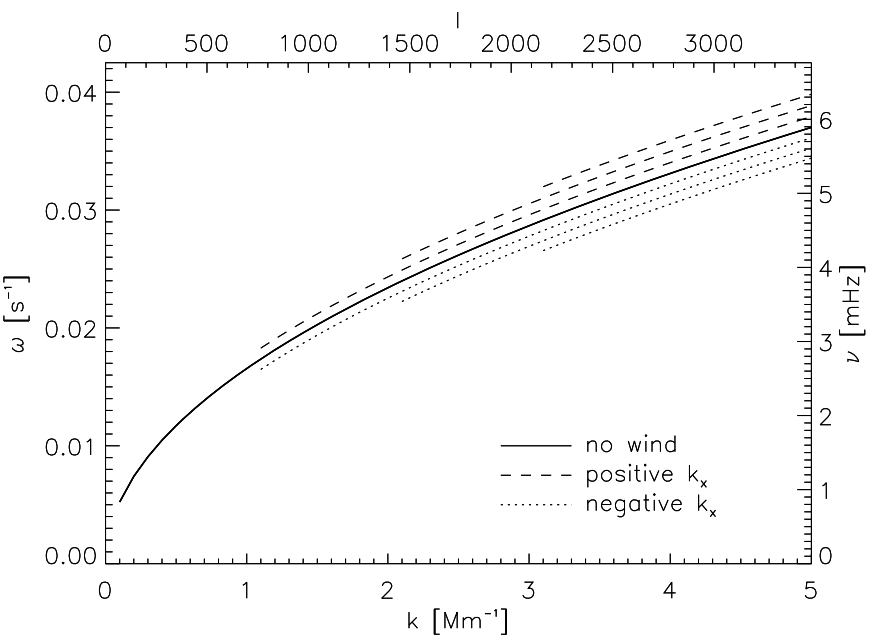

Fig. 1. The ridges of the fundamental-mode for $k_{x}= \pm 1, \pm 10$, $\pm 100 \mathrm{Mm}^{-1}$.

Inserting $\kappa$ and $\mu$, we finally obtain the dispersion relation:

$\mp \frac{1}{2 \lambda^{2}} A \sqrt{(A-1)^{2}-4 C}+\frac{1}{4 \lambda^{2}}+\frac{3 A^{2}}{4 \lambda^{2}}$

$-A\left[\frac{1}{2 \lambda^{2}}+\frac{1}{2 \lambda}\right]-\frac{1}{\lambda^{2}} C$

$-\frac{\Lambda^{2}}{\lambda^{2}}\left[\frac{D^{2}}{\lambda^{2}}+\frac{B^{2} A^{2}}{4 \lambda^{2}}+B^{2}\left[\frac{1}{2}-\frac{1}{2 \lambda}\right]^{2}\right.$

$\left.-\frac{A B D}{\lambda^{2}}-\frac{D B}{\lambda}\left[1-\frac{1}{\lambda}\right]+\frac{A B^{2}}{2 \lambda}\left[1-\frac{1}{\lambda}\right]\right]$

$+\frac{2 \Lambda}{\lambda}\left[\frac{D}{\lambda}-\frac{B A}{2 \lambda}-B\left[\frac{1}{2}-\frac{1}{2 \lambda}\right]\right]\left(j+\frac{1}{2}\right)$

$-\left(j+\frac{1}{2}\right)^{2}=0$.

This equation is solved numerically. For $v=0$ it reduces to the dispersion relation given by Schmitz \& Steffens 1999. For the sun, we take a velocity corresponding to solar rotation at mid latitudes: $v=2 \pi 6.9610^{10} /(28 \times 86400) \mathrm{cm} / \mathrm{s}=1.8 \times 10^{5} \mathrm{~cm} / \mathrm{s}$. For special cases as $\lambda=1$ or $\gamma \rightarrow \infty$ there is no simplification of the relation. Only two cases are simple, that of the f-mode and that of the homogeneous gas, which will be discussed in the following two sections.

\section{The f-mode}

From Eqs. (7) and (8), for $\Delta p=0$, we obtain the fundamental mode

$\left(\omega-k_{x} v\right) \omega=k g$.

The solution reads:

$\omega=\sqrt{g k+v^{2} k_{x}^{2} / 4}+v k_{x} / 2$.

Figure 1 shows ridges of the f-mode for $v=0$ and for the solar rotational velocity for various $k_{x}$. 


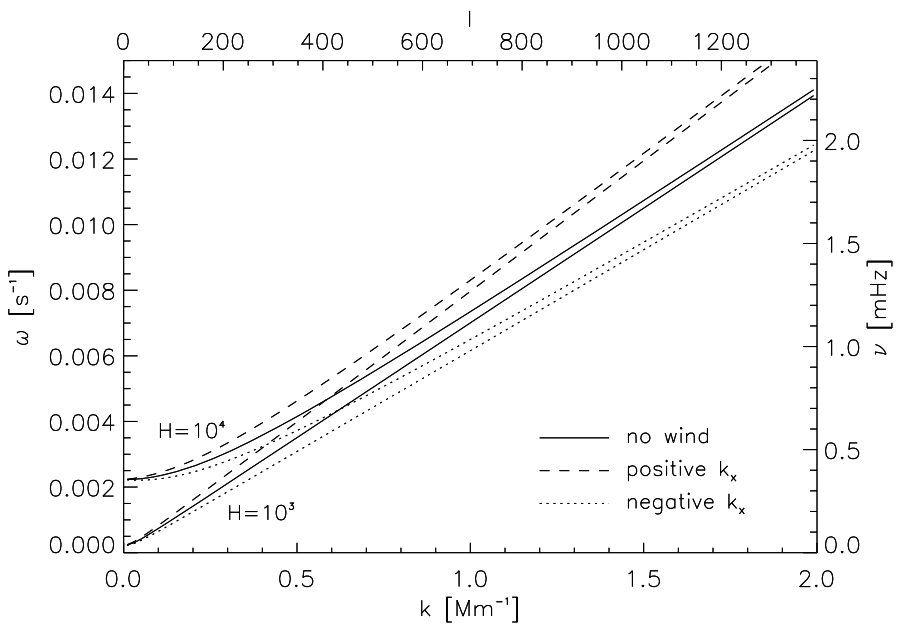

Fig. 2. Ridges of the modes of the homogeneous gas for $H=$ $10^{3} \mathrm{~km}$ and $H=10^{4} \mathrm{~km}$ and $k_{x}=0.1 \mathrm{Mm}^{-1}$.

\section{The homogeneous gas}

As this case is certainly not new we shall be brief. We need it only for a comparison and the explanation of the behavior of the stellar modes. The wave equation of the displacement $\xi$ reduces to

$\frac{\mathrm{d}^{2} \xi}{\mathrm{d} z^{2}}+\frac{1}{c_{0}^{2}}\left[\omega^{2}-k^{2} c_{0}^{2}-v k_{x} \omega\right] \xi=0$.

The solution is:

$\xi=\sin \left(k_{z} z\right)$

with

$k_{z}=\frac{1}{c_{0}} \sqrt{\omega^{2}-k^{2} c_{0}^{2}-v k_{x} \omega}$.

We put boundary conditions

$\xi=0 \quad$ at $\quad z=0$ and $z=\pi H$

to obtain

$\omega^{2}-v k_{x} \omega-\left(k_{x}^{2}+k_{y}^{2}\right) c_{0}^{2}-c_{0}^{2} \frac{1}{\pi^{2} H^{2}}=0$.

The solution is:

$\omega=\frac{1}{2}\left[v k_{x}+\sqrt{k_{x}^{2}\left(v^{2}+4 c_{0}^{2}\right)+4 c_{0}^{2} k_{y}^{2}+c_{0}^{2} \frac{4}{\pi^{2} H^{2}}}\right]$.

Figure 2 shows the frequency $\omega$ as a function of $k$ for $H=$ $10^{3} \mathrm{~km}$ and $10^{4} \mathrm{~km}$.

The range of the amplitudes of the f-mode extends from $z=$ $-\infty$ to $z=+\infty$. Accordingly all ridges are different.

On the other hand, the range of the oscillations of the homogeneous gas depends on the height $H$. With increasing height, up to $H \approx 10^{4} \mathrm{~km}$ the differences of the ridges increase. Above $H \approx 10^{4} \mathrm{~km}$, there are no differences.

\section{The Sun}

For the Sun we studied the cases $n=3 / 2$ and $n=3$ for various values of $k_{x}$. However, before looking at the results for the effects of rotation, let us first compare the eigenmodes of our idealized model with a real solar model and actual observations. Figures 3

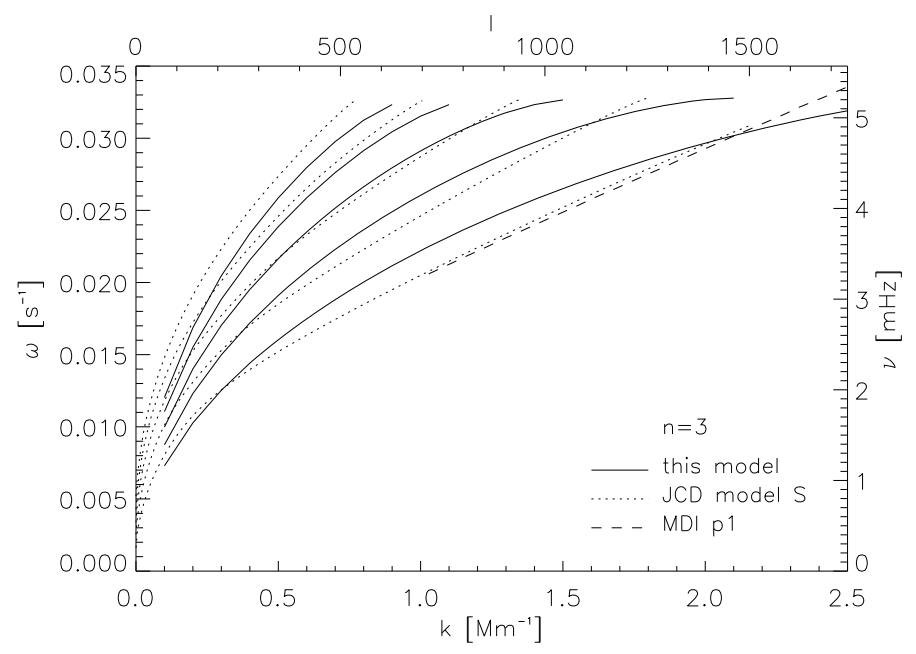

Fig. 3. Comparison of the eigenmodes $\mathrm{p} 1$ to $\mathrm{p} 5$ of our analytical model (solid lines) for $n=3$ with those of model S of Christensen-Daalsgard et al. (1996) (dotted lines) and high-degree observations of p1 by SOHO/MDI (dashed line; from Duvall et al. 1998).

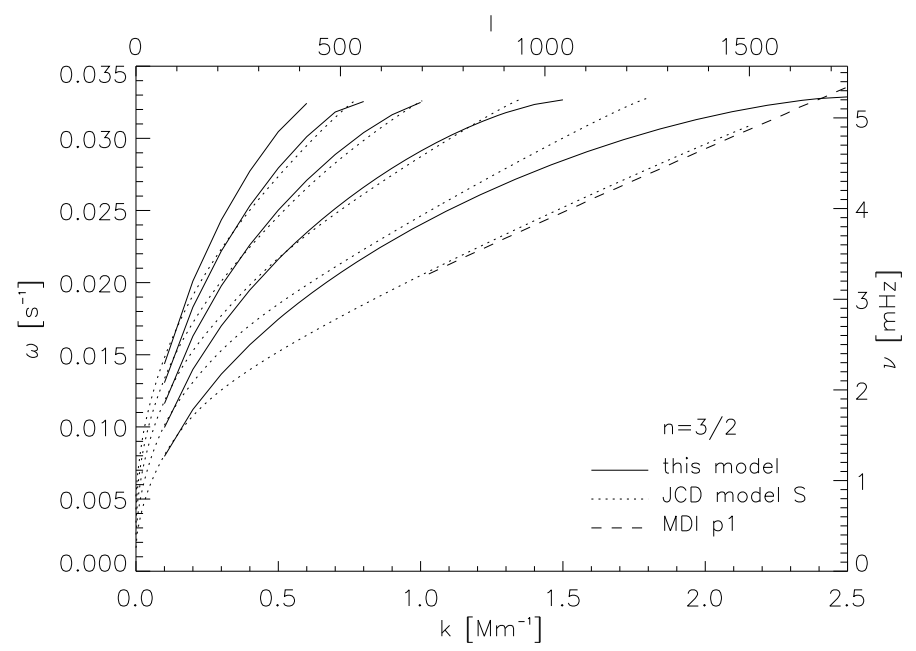

Fig. 4. Same as Fig. 3 but for $n=3 / 2$.

and 4 show comparisons of the p1 to p5 modes of our analytical model with those of model $\mathrm{S}$ of Christensen-Dalsgaard et al. (1996) and the high-degree extension of the p1 mode as observed by SOHO/MDI (Duvall et al. 1998).

While one might claim that the mode structure is qualitatively similar in the two models, quantitatively there are considerable differences, which is not surprising. The differences between our analytical model and model $\mathrm{S}$ are actually bigger than the effects of rotation which are discussed below. Figures 3 and 4 demonstrate the limitations of our idealized model for direct comparisons with current observations. It clearly lacks essential aspects of the complex physics of the sun. It is interesting to note that the differences between the two models are smaller for $n=3$ (Fig. 3) than for $n=3 / 2$ (Fig. 4). The case $n=3$ apparently is a better approximation to the real sun. This is probably because $n=3 / 2$ or $\Gamma=5 / 3$ is the index of a neutral gas, whereas $n=3$ or $\Gamma=4 / 3$ is nearer to $\Gamma \approx 1.2$ due to ionization.

While analytical studies are more limited than numerical studies because of the necessary simplifications and approximations, they provide complimentary information that is useful for developing a fundamental understanding of the basic physical problems at hand. 


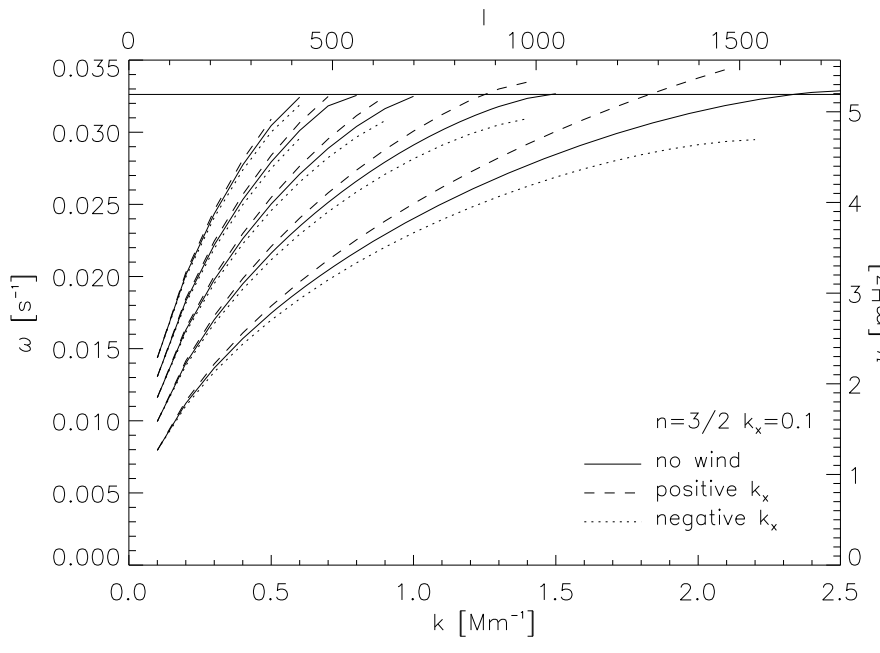

Fig. 5. Ridges of the modes for $n=3 / 2$ and $k_{x}=0.1 \mathrm{Mm}^{-1}$.

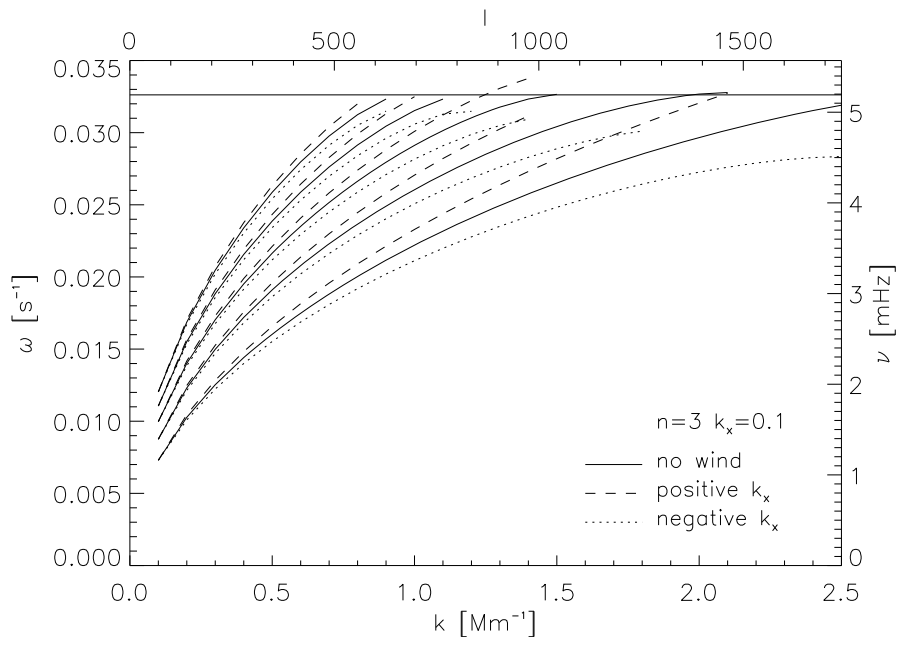

Fig. 6. Ridges of the modes for $n=3$ and $k_{x}=0.1 \mathrm{Mm}^{-1}$.

Figures 5 and 6 show the p1 to p5 modes without rotation and with solar rotation at mid latitudes for $k_{x}= \pm 0.1 \mathrm{Mm}^{-1}$, for $n=3 / 2$ and $n=3$, respectively. These are cuts through the $k_{x}-k_{y}-\omega$ cube at fixed $k_{x}$. As expected, the ridges for positive $k_{x}$ are above, the ridges for negative $k_{x}$ below the ridges of the windless case. With increasing $k_{x}$, the differences of the ridges increase. However, there are no more differences for $k_{x}>1 \mathrm{Mm}^{-1}$. The reason is that the range of oscillations with $l>1000$ is restricted to the outer convection zone and the atmosphere. Therefore, as for the homogeneous gas, there are no differences for higher values of $k_{x}$.

The findings can be compared to observed high-resolution $k-\omega$ diagrams as presented e.g. by Mitra-Kraev et al. (2008). The observed broadening of the ridges of Fig. 1 in their paper corresponds to the broadening of the ridges of Figs. 1, 3, and 4 in this paper. In both cases, the f-mode shows the strongest effects.

\section{Conclusions}

We presented a simple analytic model of a convection zone with an overlying isothermal atmosphere. As regards the representation of the dispersion relation $F(\omega, k)=0$, this model does not have the shortcomings of two-layer models. For the new model the three-dimensional adiabatic wave equation can be solved analytically by reduction to Whittaker's differential equation. The dispersion relation is an algebraic equation, which is solved numerically. We presented dispersion curves of acoustic modes with $\gamma \neq 1+1 / n$ and of modes with $\gamma=1+1 / n$. Two special cases were studied separately: the f-mode and the homogeneous gas. The model can be used for further investigations; for example, to study the problem of the generation of the observed ridges above the acoustic cut-off frequency, the existence of modes with complex frequencies, and also gravity modes. As for the simple polytropic layer, the amplitudes of the modes are given in terms of Whittaker functions. The mathematical procedure is similar to the procedure used for the simple polytropic layer.

Acknowledgements. We thank an anonymous referee for useful comments, and Jørgen Christensen-Daalsgard for providing a table of the eigenfrequencies of his standard solar model.

\section{References}

Ando, H., \& Osaki, Y. 1977, PASJ, 29, 221

Antja, H. M., \& Basu, S. 1999, ApJ, 519, 400

Abramowitz, M., \& Stegun, I. A. 1965, Handbook of Mathematical Functions (New York: Dover Publ. Inc.)

Beer, T. 1975, Atmospheric Waves (London: Adam Hilger)

Carlson, M., \& Stein R. F. 1995, ApJ, 440, L29

Christensen-Daalsgard, J. 1980 MNRAS, 190. 765

Christensen-Daalsgard, J., Däppen, W., Ajukov, S. V., et al. 1996, Science 272, 1286

Deubner, F.-L. 1975, A\&A, 44, 371

Deubner, F.-L., \& Gough, D. 1984, ARA\&A, 22, 593

Deubner, F.-L., Ulrich, R. K., \& Rhodes, E. J. 1979, A\&A, 72, 177

Duvall, T. L., Kosovichev, A. G., \& Murawski, K. 1998, ApJ, 505, L55

Lamb, H. 1932, Hydrodynamics (Cambridge: University Press)

Ledoux, P., \& Walraven, Th. 1958, Handbuch der Physik 51, 528 ed.: S. Flügge (Berlin: Springer-Verlag)

Lynden-Bell, D., \& Ostriker, J.-P. 1967, MNRAS, 136, 293

Mitra-Kraev, U., Kosovichev, A. G., \& Sekii, T. 2008, A\&A, 481, L1

Saio, H. 1993, ASS, 210, 61

Schmitz, F. 1984, A\&A, 131, 309

Schmitz, F., \& Fleck, B. 1994, A\&AS, 106, 129

Schmitz, F., \& Steffens, S. 1999, A\&A, 344, 973

Schou, J., Antia H. M., Basu, S. et al. 1998, ApJ, 505, 390

Steffens, S., \& Schmitz, F. 2000, A\&A, 354, 280

Tassoul, J.-L. 1978, Theory of Rotating Stars (Princeton: Princeton University Press) 